\title{
Naming the unnamed: Over 45,000 Candidatus names for unnamed Archaea and Bacteria in the Genome Taxonomy Database
}

MarkJ.Pallen ${ }^{1,2,3}[$ orcid.org/0000-0003-1807-3657],

Nabil-Fareed Alikhan² [orcid.org/0000-0002-1243-0767]

1 Norwich Medical School, University of East Anglia, Norwich Research Park, Norwich, Norfolk, United Kingdom

${ }^{2}$ Quadram Institute Bioscience, Norwich Research Park, Norwich, Norfolk, United Kingdom

${ }^{3}$ School of Veterinary Medicine, University of Surrey, Guildford, Surrey, United Kingdom

\section{${ }^{*}$ Corresponding author}

m.pallen@uea.ac.uk

\section{Keywords}

Bacterial nomenclature; archaeal nomenclature; genome taxonomy; shotgun metagenomics; Candidatus names 


\section{Abstract}

Thousands of new bacterial and archaeal species and higher-level taxa are discovered each year through the analysis of genomes and metagenomes. The Genome Taxonomy Database (GTDB) provides hierarchical sequence-based descriptions and classifications for new and as-yet-unnamed taxa. However, bacterial nomenclature, as currently configured, cannot keep up with the need for new well-formed names. Instead, microbiologists have been forced to use hard-to-remember alphanumeric placeholder labels. Here, we exploit an approach to the generation of well-formed arbitrary Latinate names at a scale sufficient to name tens of thousands of unnamed taxa within GTDB. These newly created names represent an important resource for the microbiology community, facilitating communication between bioinformaticians, microbiologists and taxonomists, while populating the emerging landscape of microbial taxonomic and functional discovery with accessible and memorable linguistic labels.

\section{Data summary}

Input files for this study were obtained from the following sources

- Whitaker's Latin stems: http://archives.nd.edu/whitaker/wordsall.zip

- English Wiktionary headwords: https://dumps.wikimedia.org/enwiktionary/20210920/enwiktionary-20210920-pages-articlesmultistream-index.txt.bz2

- genus names compiled by Global Biodiversity Information Facility: https://hosteddatasets.gbif.org/datasets/backbone/backbone-current-simple.txt.gz

- GDTB metadata and taxonomy files:

https://data.gtdb.ecogenomic.org/releases/release202/202.0/

- Zenodo files: https://zenodo.org/record/5652886, includes input files ar_genus_endings.txt, bac_genus_endings.txt and species_endings.txt and all output files specified in the manuscript and the shell script GTDB_renamer.sh.

Python scripts and the GTDB_renamer.sh shell script used in this analysis are available on GitHub

Supplementary files:

- https://github.com/quadram-institute-bioscience/namingGTDB

- Table S1 Creation and use of well-formed arbitrary, meaningless Latinate names in naming Archaea and Bacteria (Excel file with multiple tabs, carrying data from output files in the Zenodo archive)

- Table S2 New names for Archaea and Bacteria

- File S1 Archaeal Protologues (Word file).

- File S2 Bacterial Protologues (Word file).

- File S3 Renamed GTDB taxonomy file for use with the GTDB Toolkit (.tsv file) 


\section{Introduction}

We microbiologists live in an age of genomic plenty [1]. Genome and metagenome analyses have fuelled exponential growth in the identification of new taxa of Archaea and Bacteria [2]. In addition, ready availability of genome sequences has primed the development of comprehensive sequencebased taxonomies, such as the Genome Taxonomy Database (GTDB) [3, 4]. However, such exhilarating success in discovering and classifying new microorganisms has created an urgent new challenge: how are we going to name all these newfound microbial taxa?

Linnaean binomials, typically drawing on combinations of Latin and Ancient Greek roots, have stood the test of time in providing a stable, clear and memorable system of nomenclature across the tree of life $[5,6]$. However, in the absence of mechanisms for the speedy creation of well-formed Latin names at scale, the GTDB team has adopted a system of alphanumeric placeholders for newly delineated taxa, which are hard to remember and are easily confused. The current system includes alphanumerical species epithets (e.g. sp011333035) derived from numerical identifiers of representative genomes and alphanumeric designations for genera and higher-level taxa (e.g. CG2-3070-394), selected arbitrarily from identifiers in public sequence databases. These unnamed placeholder taxa now clearly outnumber those with names: in the latest release of the GTDB taxonomy [7] around a quarter of species (12,398 or 25.9\%) have been assigned Latin names, while roughly three quarters of the species $(35,496$ or $74.1 \%$ ) are identified only by alphanumeric placeholders labels.

Nomenclature of Archaea and Bacteria is governed by The International Code for Nomenclature of Prokaryotes (the ICNP) [8]. Most names for Archaea and Bacteria have been applied to taxa that can be maintained in stable culture. However, since the 1980s, there has been a growing recognition that uncultured taxa can nonetheless be identified and classified via analysis of macromolecular sequences [9-11]. To accommodate such uncultured taxa, Murray and Schleifer proposed a new category of taxonomic names, which they termed Candidatus [12]. Recommendations on the use of the category Candidatus are now included in the ICNP, alongside clarification that such names are provisional and enjoy no standing in nomenclature. In a recent review, one of us (Pallen) concluded that the category Candidatus continues to serve a useful function in providing well-formed names for uncultured taxa [13].

Most names for taxa of Archaea and Bacteria are descriptive or named after people or places. However, the use of arbitrary names has a long history in taxonomy and is clearly sanctioned within the ICNP $[8,14]$. With these considerations in mind, here we address the challenge of "naming the unnamed" by creating nearly 60,000 arbitrary well-formed Latin names for Archaea and bacteria and then using a subset of them to assign Candidatus names to all unnamed taxa in GTDB.

\section{Methods}

\section{Creation of arbitrary Latinate names}

The workflow used to create and assign arbitrary Latinate names is shown in Figure 1. The workflow can be run using the Linux shell script GTDB_renamer.sh. To ensure selection of initial wordcomponents consistent with the phonotactics of Latin (the rules governing how sounds are combined to create syllables), a publicly available set of Latin stems compiled by the Latinist William Whitaker was downloaded as STEMLIST.GEN from http://archives.nd.edu/whitaker/wordsall.zip in September 2021.

Page 3 of 15

preprints-51936-manuscript 
The Python script stemlist_clean.py was used to extract the relevant column from the file, to convert all entries to lower case and to remove duplicates. The resulting output file Whitakers_stems.txt was used as input for the Python script openings_creator.py. This script created a de-replicated set of five-letter Latin opening word-components that ended in consonants. The script excluded strings if they

- contained tandem vowels or difficult-to-pronounce consonant clusters

- contained word components that might carry unwanted connotations (e.g. vulgarities)

- began or ended with the letters j, k, w, y, z, absent from the original Latin alphabet.

To ensure that the opening word components used in name formation were free of meaning, the list was purged of entries identical to $\sim 6$ million headwords in the English Wiktionary, which include words from English, Latin and many other languages. In addition, the script excluded opening wordcomponents identical to genus names already in use in taxonomy, by searching against a set of unique genus names compiled by the Global Biodiversity Information Facility. Wiktionary headwords were obtained from an index file downloaded in September 2021 from https://dumps.wikimedia.org/enwiktionary/20210920/enwiktionary-20210920-pages-articles-

multistream-index.txt.bz2. Headwords were extracted from this file using the Python script enwiki_clean.py to give a file enwiki_terms.txt containing sorted de-replicated words in lowercase alphabetical characters. The entries in the file GBIF_clean.txt were extracted using a Python script GBIF-clean.py from the file downloaded via https://hosteddatasets.gbif.org/datasets/backbone/backbone-current-simple.txt.gz. The files enwiki_terms.txt and GBIF_clean.txt were concatenated into a file excluded_terms.txt, which was used by the openings_creator.py script.

To select final word components for names that comply with phonotactic and grammatical norms of Latin, a set of distinctive feminine Latin suffixes was selected from a list compiled by Wikipedia (https://en.wiktionary.org/wiki/Category:Latin feminine suffixes), alongside short nouns suitable for use as non-specific descriptors of microbes (Table 1). For ease of use, these final word components have been restricted to the feminine gender. To facilitate the use of genus names in creating names of higher-level taxa by addition of suffixes to the stem, five short final word-components belonging to the first declension were selected for use in bacterial genus names and incorporated into the file bac_genus_endings.txt. The single final word component archa (derived via Latinisation of the Greek

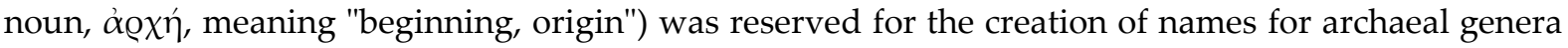
and deployed in the file ar_genus_endings.txt. The remaining final word-components, which belong to the first and third declensions, were incorporated into the file species_endings.txt.

The Python script name-creator.py was run three times to combine all opening word-components with all final word-components in the bac_genus_endings.txt, ar_genus_endings.txt and species_endings.txt files to create the output files bac_genus_names.txt, ar_genus_endings.txt and species_names.txt. As with the

\section{Assignment of arbitrary names to unnamed taxa}

The Python script, name_table_maker.py was used to extract all alphanumeric taxon names from the latest versions of the GTDB taxonomy files for Archaea and Bacteria (ar122_taxonomy_r202.tsv and bac120_taxonomy_r202.tsv) and to sort them by rank in the taxonomic hierarchy (i.e. listing phylum designations before class designations etc). The script used the archaeal_genus_names.txt, bacterial_genus_names.txt and species_names.txt files as input and after randomizing the order of the genus names and species names and then sorting genus names by simplicity (deploying those with no double consonants first), the script assigned Latin names to the unnamed taxa Archaea and Bacteria identified in the GTDB taxonomy files.

Page 4 of 15

preprints-51936-manuscript 
The Python script taxon_renamer.py was then used to replace all alphanumeric designations with Latinate names in the GTDB taxonomy and metadata files for Archaea and Bacteria to create taxonomy files suitable for use with the GTDB toolkit [15], together with metadata files providing relevant details on the newly named taxa. New names were marked by the addition of an exclamation mark so that they could be easily distinguished from existing names in the taxonomy and metadata files. However, unmarked versions of the files were created suitable for use by the GTDB toolkit in assigning genomes to taxa.

The Python scripts archaeal_protologue_maker.py and bacterial_protologue_maker.py were used to output relevant descriptive information from the renamed GDTB metadata files in Rich Text Format (RTF) to create protologues suitable for publication of Candidatus names for use in the scientific literature and in public databases.

\section{Results}

\section{Creation of arbitrary Latinate names}

After extracting all unique initial five-letter-strings from the Latin stems compiled by William Whitaker, excluding unwanted clusters of letters and ensuring that all the strings did not form meaningful words in English, Latin and other major languages, we were left with 1,474 suitable opening word-components (Table S1). When these were combined with our curated set of final wordcomponents used in Latin-while taking care to exclude terms with pre-existing meanings or genus names already used in taxonomy-we created 1,473 arbitrary, meaningless names for archaeal genera, 9,970 arbitrary, meaningless names for bacterial genera and 48,530 species epithets suitable for naming species from either domain (Table S1).

\section{Assignment of arbitrary names to unnamed taxa}

After retrieving 42,000 alphanumeric placeholder names from the GTDB taxonomy files, we built name-replacement tables showing the placeholder names alongside replacement well-formed Latin names (Table S1). Chuvochina et al have emphasized the importance of specifying type material for uncultured taxa [16]. GTDB makes an arbitrary choice as to which placeholder labels should be propagated from the level of genus up to higher taxa, which, in turn, specifies which genus should be considered to act as type genus for those higher taxa. We respected the choices made by GTDB for type genera, replacing alphanumeric designations with arbitrary names wherever they occur in the taxonomy hierarchy, using the new name for each type genus to build names by addition of suffixes specified in the ICNP and elsewhere $[8,17]$.

Following the GTDB's lead in making an arbitrary choice of type material, we made an arbitrary choice as to which species should be designated the type species for a previously unnamed genus. GTDB also adopts the approach of giving each unnamed species a unique alphanumeric designation. Although, under the rules of the ICNP, there is no need for each species epithet to be unique, for simplicity and consistency, we have followed GTDB's practice and assigned a unique species epithet to each of the unnamed species in the current database.

The name-replacement tables were used to replace placeholders with the new names in the GTDB taxonomy and metadata files. In so doing, we assigned 650 new archaeal genus names and 1,806 new archaeal species names along with 8,607 new bacterial genus names and 31,072 new bacterial species names. Unused genus names and species epithets were retained for use with later releases of the database. When the new genus names were used to build new names for higher-level taxa, we created new names for 42 bacterial and 4 archaeal phyla (Table 2); 14 archaeal classes, 64 archaeal orders and 251 archaeal families; plus 2050 bacterial families, 749 bacterial orders and 188 bacterial classes. For 320 taxa, GTDB uses placeholders for higher-level taxa that have not been applied to a genus. In these

Page 5 of 15

preprints-51936-manuscript 
cases, we have replaced the placeholders with new Latinate names in the taxonomy files, but have left these out of the protologues, as we anticipate that these anomalies will be addressed in subsequent releases of the GTDB.

Although the renamed GTDB metadata files contain a rich set of descriptive data for the newly named taxa, mindful that some authorities prefer to see descriptions of new taxa in a text-based format, we have created two RTF files - a file format that can be opened by most word processors containing protologues for all the new Candidatus taxa (File S1, File S2). However, as these traditional protologues take up over 8,000 pages, they cannot be presented in the main body of this manuscript. The GTDB_renamer.sh script also created tables of new names from these protologue files, with the new names ordered by taxonomic rank (phyla shown in Table 2, all taxa shown in Table S2). We confirmed that the renamed GTDB taxonomy file (File S3) worked as expected by running the GTDB toolkit over a set of metagenome-associated genomes from the horse gut (data not shown). This required replacing the original taxonomy file used by the GTDB Toolkit with renamed GTDB taxonomy file, while retaining the original file name expected by the program, gtdb_taxonomy.tsv.

\section{Discussion}

Conventional approaches to the creation of new names for Archaea and Bacteria generally rely on descriptive names or names associated with people or places. Over the last ten years, such approaches have delivered around a thousand new validly published species names per year and tens-tohundreds of Candidatus names annually [13, 18]. However, given a backlog of $>32,000$ well classified but unnamed species in GTDB, using conventional approaches at the current rate of progress would take at least thirty years to name all these unnamed taxa - by which time, we would be faced with the problem of naming hundreds of thousands more newly discovered species! Recently, Pallen et al [19] described an approach enabling automated creation of descriptive names en masse. However, deployment of functionally descriptive names at the scale needed here would require reconstruction of the phenotypic properties of tens of thousands of species, which is a non-trivial and error-prone task. Similarly, assigning names based on habitat requires exhaustive searches of genome metadata to ensure names are accurate and precise. In addition, there is a trade-off here between the semantic specificity of a descriptive name and its length and usability, as is evident from recently proposed names such as Hoministercoradaptatus ammoniilyticus, Anthropogastromicrobium aceti and Porcipelethomonas ammoniilytica [20].

Here, given the failure of conventional approaches, we have taken what might seem like a radical step in creating and applying arbitrary well-formed Latinate names to unnamed uncultured taxa of Archaea and Bacteria. However, formation of names in an arbitrary fashion has a long tradition in taxonomy. Linnaeus created arbitrary names via anagrams, e.g. the genus name Mahernia as an imperfect anagram of Hermannia [6]. In the 1830s, the eminent English botanist John Lindley wrote: "So impossible is it to construct generic names that will express the peculiarities of the species they represent, that I agree with those who think a good, well-sounding, unmeaning name as good as any that can be contrived" [21]. Soon after, Scottish naturalist George Johnston created the arbitrary genus name Carinella for a marine annelid [22].

In 1869, in his groundbreaking Lois de Nomenclature Botanique [23], the Swiss botanist Alphonse De Candolle wrote: "Generic names are drawn from certain characters, from certain appearances... and even from combinations of letters that are quite arbitrary. All that is required of a name is that it shall lead neither to confusion nor to error..." Early 20th-century biologist William Kearfott created over a hundred arbitrary rhyming species epithets-e.g. bana, cana, dana; bobana, cocana, dodana; boxcana, coxcana, doxcana - many of which still belong to validly published names in use today [24].

Page 6 of 15

preprints-51936-manuscript 
In 1952, palaeobiologist Raymond Casey invented the Greek-sounding name Gythemon for an extinct clam, cited in the Zoological Code as a name built from 'an arbitrary combination of letters' [25]. A few years later, the botanist Gordon Rowley professed that "names are more often than not mere handles, and the most that we can ask of a handle is that it be neat and easy to grasp". The current version of the ICNP also makes clear "The primary purpose of giving a name to a taxon is to supply a means of referring to it rather than to indicate the characters or the history of the taxon" and names "may... be composed in an arbitrary manner" [8].

In fact, there are many precedents for the arbitrary formation of names in bacterial nomenclature, including names derived from organizational acronyms, such as Cedecea (from CDC), names derived in an arbitrary fashion from personal names, such Simkania (after Simona Kahane) or names created from arbitrary contractions of functional descriptions, such as Methermicoccus [14].

Principle 3 of the ICNP [8] states "scientific names of all taxa are Latin or latinized words treated as Latin regardless of their origin". In English, Latinate terms form a lexical stratum with a distinctive phonotactic "Latinity", associated with scientific or scholarly writing [26, 27]. Here, to comply with the requirement of the ICNP, while also respecting the look and feel of the language, we have used words or syllables derived from Latin as components of new names. The result is a set of names that recall the familiarity and gravitas of Latin, even though they are devoid of etymology or meaning.

According to the strictest interpretation, Candidatus names can be assigned only to uncultured taxa and, here, we have made the assumption that all placeholder names in GTDB are associated with uncultured taxa. However, as Pallen [13] has argued elsewhere, even if a small number of the renamed taxa do already have cultured representatives, we can safely fall back upon the broader definition of Candidatus as a category 'used for describing prokaryotic entities ... for which characteristics required for description according to the Code are lacking'.

The fact that Candidatus names have no standing in nomenclature is often seen as a deficiency of the current system of bacterial nomenclature [28]. However, the provisional status of the names proposed here can be seen as helpful, in that if, in the future, those working on a given taxon wish to propose a new name, they are perfectly entitled to do so within the current system - albeit with the proviso that the opening principle of the ICNP is to "aim for stability in names". Aside from such changes, the vast majority of the Candidatus names proposed here are likely to remain highly stable-given that only $0.26 \%$ of genomes are on average assigned to a different species cluster from one release of GTDB to the next [7]- and so can now be safely adopted by databases and used in the scientific literature.

The scale of our efforts here, together with the fact that we are naming taxa that have already been delineated and classified by others, begs the question "Who has the right to create and assign taxonomic names?" Although the ICNP says nothing on this issue, traditionally the task of naming new cultured species has fallen to those who isolate and discover the species and deposit type material in culture collections. As this often requires a lot of work, the act of naming can be seen as a reward for the "sweat of one's brow". However, it remains unclear whether similar principles can be applied to the naming of uncultured species defined only by sequence analysis, when who can say who should be rewarded with a stake in the process: those who collected samples, those who sequenced them, those who binned reads into metagenome-assembled genomes or those who performed the sophisticated phylogenetic analyses delineating and classifying uncultured taxa? In any case, so far, none of these parties has shown interest in-or developed competing methodologies for-creating new names at scale.

Page 7 of 15

preprints-51936-manuscript 
It is worth stressing that the age of microbial discovery is far from over. Each new GTDB release is going to bring a fresh deluge of new taxa needing new names. Fortunately, additional names remain available from the set created here, which can be used in the near future. Further ahead, new names can be created following the principles established here with only minor changes to procedures or input files (e.g. including Greek stems, longer initial word-components or additional final wordcomponents).

Drawing on the encouraging precedent with new names for SARS variants of concern [29], we hope that the names proposed here are rapidly adopted by the scientific community and used widely. After all, the alternative remains continuing use of confusing, hard-to-remember alphanumeric designations into the indefinite future. Instead, we propose a system of bacterial nomenclature fit for the age of genomics and ambitious enough to cope with the exciting discoveries yet to come.

\section{Author statements}

\section{Authors and contributors}

Conceptualization, MJP; Data curation, Methodology: MJP and NFA; Writing: MJP.

\section{Conflicts of interest}

The authors declare that there are no conflicts of interest.

\section{Funding information}

MJP is supported by the Quadram Institute Bioscience BBSRC-funded Strategic Program: Microbes in the Food Chain (project no. BB/R012504/1) and its constituent project BBS/E/F/000PR10351 (Theme 3, Microbial Communities in the Food Chain) and by the Medical Research Council CLIMB-BIG-DATA grant MR/T030062/1. NFA is supported by the Quadram Institute Bioscience BBSRC funded Core Capability Grant (project number BB/CCG1860/1).

\section{Acknowledgements}

We thank Andrea Telatin for help in creating the first version of the taxon_renamer.py Python script. We thank Phil Hugenholtz for providing feedback on an early draft of the manuscript. We thank Rachel Gilroy for confirming that the renamed GTDB taxonomy files worked as expected with the GTDB Toolkit. 


\section{Figures and tables}

\section{Table 1 Final word-components used to create arbitrary names}

The feminine Latin suffixes were selected from a list compiled by Wikipedia (https://en.wiktionary.org/wiki/Category:Latin feminine suffixes) and were used alongside short nouns suitable for use as non-specific descriptors of microbes. The resulting names, were all defined as feminine Neo-Latin nouns, which when used as species epithets, are deployed as nouns used in apposition.

\begin{tabular}{|c|c|c|c|}
\hline $\begin{array}{l}\text { Component } \\
\text { (nom., gen.) }\end{array}$ & Declension & Application & Derivation \\
\hline archa, archae & 1 & Archaeal genera & Latinised derivative of Gr. fem. n. ápxń, beginning, origin \\
\hline ana, anae & 1 & Bacterial genera & Latin suffix to a noun stem to form an adjective \\
\hline aria, ariae & 1 & Bacterial genera & Latin suffix used to form abstract nouns from other nouns \\
\hline ella, ellae & 1 & Bacterial genera & Latin suffix used to form a diminutive of a noun \\
\hline ia, iae & 1 & Bacterial genera & Latin suffix used to form an abstract noun \\
\hline osa, osae & 1 & Bacterial genera & Latin suffix used to form adjectives from nouns meaning "full of" \\
\hline ula, ulae & 1 & Bacterial genera & Latin suffix used to form a diminutive of a noun \\
\hline astra, astrae & 1 & Species & Latin suffix of nouns, expressing resemblance \\
\hline atica, aticae & 1 & Species & Latin suffix used to form adjectives indicating a relation to the root noun \\
\hline entia, entiae & 1 & Species & Latin suffix used to form an abstract noun \\
\hline etta, ettae & 1 & Species & Latin suffix used to form a diminutive of a noun \\
\hline ibra, ibrae & 1 & Species & Latin suffix of nouns denoting instrument, vessel, place or person \\
\hline ibula, ibulae & 1 & Species & Latin suffix of nouns denoting instrument, vessel, place or person \\
\hline icella, icellae & 1 & Species & Connecting vowel -i-; L. fem. n. cella, a cell \\
\hline icula, iculae & 1 & Species & Latin suffix used to form a diminutive of a noun \\
\hline ifica, ificae & 1 & Species & Latin suffix forming adjectives that denote bringing or making. \\
\hline iforma, iformae & 1 & Species & Connecting vowel -i-; L. fem. n. forma, a form \\
\hline igena, igenae & 1 & Species & Latin suffix meaning "born from, sprung form" \\
\hline ilega, ilegae & 1 & Species & Latin suffix forming adjectives related to the concept of collecting \\
\hline ilenta, ilentae & 1 & Species & Latin suffix forming adjectives meaning "abounding in, full of" \\
\hline imonia, imoniae & 1 & Species & Latin suffix used to form abstract nouns from adjectives \\
\hline isca, iscae & 1 & Species & Late Latin suffix used to form adjectives \\
\hline issa, issae & 1 & Species & Late Latin suffix used to form feminine forms of masculine nouns. \\
\hline itia, itiae & 1 & Species & $\begin{array}{l}\text { Latin suffix to form an abstract noun describing the condition of being } \\
\text { something. }\end{array}$ \\
\hline itica, iticae & 1 & Species & Latin suffix added to nouns to form adjectives \\
\hline itoga, itogae & 1 & Species & $\begin{array}{l}\text { Connecting vowel -i-; L. fem. n. toga, a covering, garment, used non- } \\
\text { specifically in many bacterial names }\end{array}$ \\
\hline itura, iturae & 1 & Species & Latin suffix used to form a noun relating to an action. \\
\hline ivita, ivitae & 1 & Species & Connecting vowel -i-; L. fem. n. vita, life \\
\hline ousia, ousiae & 1 & Species & Gr. fem. n. ousia, essence \\
\hline ago, aginis & 3 & Species & Latin suffix , forms nouns describing objects, plants, and animals. \\
\hline atio, ationis & 3 & Species & Latin suffix added to a verb to form an abstract noun \\
\hline
\end{tabular}




\begin{tabular}{|l|l|l|l|}
\hline ax, acis & 3 & Species & Latin suffix used to form adjectives expressing a tendency or inclination \\
\hline edo, edinis & 3 & Species & Latin suffix used to form abstract nouns \\
\hline imonas, imonadis & 3 & Species & Connecting vowel -i-; L. fem. $\mathrm{n}$. monas, a unit, monad \\
\hline itas, itatis & 3 & Species & Latin suffix used to form abstract nouns indicating a state of being. \\
\hline itio, itionis & 3 & Species & Latin suffix used to form a noun relating to an action. \\
\hline itrix, itricis & 3 & Species & Latin suffix used to form a feminine agent noun \\
\hline itudo, itudinis & 3 & Species & Latin suffix forming an abstract noun indicating a state \\
\hline ugo, uginis & 3 & Species & Latin suffix forms nouns denoting coating of material \\
\hline
\end{tabular}


Table 2 Newly Named Phyla

\begin{tabular}{|c|c|c|}
\hline New name & GTDB Placeholder & Domain \\
\hline Candidatus Bifararchota phyl. nov. & EX4484-52 & Archaea \\
\hline Candidatus Funivarchota phyl. nov. & SpSt-1190 & Archaea \\
\hline Candidatus Genufarchota phyl. nov. & QMZS01 & Archaea \\
\hline Candidatus Nutamarchota phyl. nov. & PWEA01 & Archaea \\
\hline Candidatus Bicoriota phyl. nov. & B130-G9 & Bacteria \\
\hline Candidatus Bifisiota phyl. nov. & GCA-001730085 & Bacteria \\
\hline Candidatus Casigariota phyl. nov. & UBA9089 & Bacteria \\
\hline Candidatus Ceracanota phyl. nov. & T1Sed10-126 & Bacteria \\
\hline Candidatus Cibaranota phyl. nov. & DUMJ01 & Bacteria \\
\hline Candidatus Ciminosota phyl. nov. & ARS69 & Bacteria \\
\hline Candidatus Cinipanota phyl. nov. & SLNR01 & Bacteria \\
\hline Candidatus Cocibosota phyl. nov. & CG2-30-53-67 & Bacteria \\
\hline Candidatus Colepanota phyl. nov. & CG2-30-70-394 & Bacteria \\
\hline Candidatus Conipanota phyl. nov. & JABMQX01 & Bacteria \\
\hline Candidatus Culilariota phyl. nov. & UBA8481 & Bacteria \\
\hline Candidatus Cululellota phyl. nov. & JACIXR01 & Bacteria \\
\hline Candidatus Cululosota phyl. nov. & FEN-1099 & Bacteria \\
\hline Candidatus Decunulota phyl. nov. & QNDG01 & Bacteria \\
\hline Candidatus Defacidota phyl. nov. & CLD3 & Bacteria \\
\hline Candidatus Defarosota phyl. nov. & DTU030 & Bacteria \\
\hline Candidatus Deforanota phyl. nov. & CAIJMQ01 & Bacteria \\
\hline Candidatus Dehinanota phyl. nov. & OLB16 & Bacteria \\
\hline Candidatus Desonellota phyl. nov. & AABM5-125-24 & Bacteria \\
\hline Candidatus Domigosota phyl. nov. & UBA10199 & Bacteria \\
\hline Candidatus Feditulota phyl. nov. & SM23-31 & Bacteria \\
\hline Candidatus Foricariota phyl. nov. & RUG730 & Bacteria \\
\hline Candidatus Funamanota phyl. nov. & UBA2233 & Bacteria \\
\hline Candidatus Lanifulota phyl. nov. & $4572-55$ & Bacteria \\
\hline Candidatus Laxitanota phyl. nov. & CG03 & Bacteria \\
\hline Candidatus Lenunidota phyl. nov. & CSSED10-310 & Bacteria \\
\hline Candidatus Levifellota phyl. nov. & SpSt-1050 & Bacteria \\
\hline Candidatus Minagiota phyl. nov. & CSP1-3 & Bacteria \\
\hline Candidatus Musulosota phyl. nov. & J088 & Bacteria \\
\hline Candidatus Nicotellota phyl. nov. & SpSt-318 & Bacteria \\
\hline Candidatus Relapellota phyl. nov. & BMS3Abin14 & Bacteria \\
\hline Candidatus Resuliota phyl. nov. & RBG-13-66-14 & Bacteria \\
\hline Candidatus Rinocanota phyl. nov. & SZUA-182 & Bacteria \\
\hline Candidatus Rubefidota phyl. nov. & JABDJQ01 & Bacteria \\
\hline Candidatus Satacellota phyl. nov. & DQWO01 & Bacteria \\
\hline Candidatus Semimosota phyl. nov. & DSWW01 & Bacteria \\
\hline Candidatus Semunulota phyl. nov. & UBA6262 & Bacteria \\
\hline Candidatus Subadanota phyl. nov. & T1SED10-198M & Bacteria \\
\hline Candidatus Televariota phyl. nov. & SpSt-956 & Bacteria \\
\hline Candidatus Venunellota phyl. nov. & UBA3054 & Bacteria \\
\hline Candidatus Volubiota phyl. nov. & RBG-13-61-14 & Bacteria \\
\hline Candidatus Bicoriota phyl. nov. & B130-G9 & Bacteria \\
\hline
\end{tabular}




\section{Figure 1}

Schematic workflow for creation and assignment of arbitrary Latinate names. Opening 5-letter strings from Latin words were selected and curated to exclude unwanted components and meaningful words. These were combined with Latin suffixes or words to create names, which were then used to name placeholder taxa in GTDB files and create text-based protologues.

\begin{tabular}{|c|c|c|}
\hline Openings Creation & $\begin{array}{l}\text { Select 5-letter opening strings from } \\
\text { Latin words }\end{array}$ & $\begin{array}{l}\text { Exclude stems with } \\
\text { unwanted components or that are } \\
\text { meaningful words }\end{array}$ \\
\hline Name Creation & $\begin{array}{l}\text { Combine stems with } \\
\text { endings to create names }\end{array}$ & $\begin{array}{l}\text { Exclude meaningful words } \\
\text { or names previously } \\
\text { used in taxonomy }\end{array}$ \\
\hline \multirow[t]{2}{*}{ Name Assignment } & \multicolumn{2}{|c|}{$\begin{array}{l}\text { Extract all alphanumeric placeholders } \\
\text { from GTDB taxonomy }\end{array}$} \\
\hline & \multicolumn{2}{|c|}{$\begin{array}{l}\text { Create name replacement tables to assign } \\
\text { well-formed arbitrary names to placeholder taxa }\end{array}$} \\
\hline Name Replacement & \multicolumn{2}{|c|}{$\begin{array}{l}\text { Replace all alphanumeric placeholders } \\
\text { from GTDB taxonomy with new arbitrary names }\end{array}$} \\
\hline Protologue Creation & Create text-based protologues $t$ & describe all newly named taxa \\
\hline
\end{tabular}




\section{References}

1. Loman NJ, Pallen MJ. Twenty years of bacterial genome sequencing. Nat Rev Microbiol. 2015;13:787-794. DOI: 10.1038/nrmicro3565

2. Parks DH, Rinke C, Chuvochina M, Chaumeil P-A, Woodcroft BJ et al. Recovery of nearly 8,000 metagenome-assembled genomes substantially expands the tree of life. Nat Microbiol. 2017;2:1533-1542. DOI: 10.1038/s41564-017-0012-7

3. Rosselló-Móra R. Towards a taxonomy of Bacteria and Archaea based on interactive and cumulative data repositories. Environ Microbiol. 2012;14:318-334. DOI: 10.1111/j.14622920.2011.02599.x

4. Parks DH, Chuvochina M, Chaumeil PA, Rinke C, Mussig AJ et al. A complete domain-tospecies taxonomy for Bacteria and Archaea. Nat Biotechnol. 2020;38:1079-1086. DOI: 10.1038/s41587-020-0501-8

5. Austin D. The Nuance and Wit of Carolus Linnaeus. The Palmetto. 1993;13:8.

6. Linnaeus C. Systema Naturae 12th Edition. Stockholm: Laurentius Salvius; 1759

7. Parks DH, Chuvochina M, Rinke C, Mussig AJ, Chaumeil PA et al. GTDB: an ongoing census of bacterial and archaeal diversity through a phylogenetically consistent, rank normalized and complete genome-based taxonomy. Nucleic Acids Res. 2021gkab776. DOI: 10.1093/nar/gkab776

8. Parker CT, Tindall BJ, Garrity GM. International Code of Nomenclature of Prokaryotes. International Journal of Systematic and Evolutionary Microbiology. 2019;69:S1-S111. DOI: DOI 10.1099/ijsem.0.000778

9. Olsen GJ, Lane DJ, Giovannoni SJ, Pace NR, Stahl DA. Microbial ecology and evolution: a ribosomal RNA approach. Annu Rev Microbiol. 1986;40:337-365. DOI: 10.1146/annurev.mi.40.100186.002005

10. Woese CR. Bacterial evolution. Microbiol Rev. 1987;51:221-271.

11. Relman DA. The identification of uncultured microbial pathogens. J Infect Dis. 1993;168:1-8. DOI: 10.1093/infdis/168.1.1

12. Murray RG, Schleifer KH. Taxonomic notes: a proposal for recording the properties of putative taxa of procaryotes. Int J Syst Bacteriol. 1994;44:174-176. DOI: 10.1099/00207713-44-1-174

13. Pallen MJ. The status Candidatus for uncultured taxa of Bacteria and Archaea: a SWOT analysis. Int J Syst Evol Microbiol. 2021;71:005000.

14. Pallen MJ. Bacterial nomenclature in the era of genomics. New Microbes and New Infections. 2021;44:100942.

15. Chaumeil PA, Mussig AJ, Hugenholtz P, Parks DH. GTDB-Tk: a toolkit to classify genomes with the Genome Taxonomy Database. Bioinformatics. 2019DOI: 10.1093/bioinformatics/btz848

16. Chuvochina M, Rinke C, Parks DH, Rappé MS, Tyson GW et al. The importance of designating type material for uncultured taxa. Syst Appl Microbiol. 2019;42:15-21. DOI: 10.1016/j.syapm.2018.07.003

17. Whitman WB, Oren A, Chuvochina M, da Costa MS, Garrity GM et al. Proposal of the suffix ota to denote phyla. Addendum to 'Proposal to include the rank of phylum in the International Code of Nomenclature of Prokaryotes'. Int J Syst Evol Microbiol. 2018;68:967-969. DOI: 10.1099/ijsem.0.002593

18. LPSN. List of Prokaryotic names with Standing in Nomenclature. 2021. https://www.bacterio.net

19. Pallen MJ, Telatin A, Oren A. The Next Million Names for Archaea and Bacteria. Trends Microbiol. 2021;29:289-298. DOI: 10.1016/j.tim.2020.10.009

20. Hitch TCA, Riedel T, Oren A, Overmann J, Lawley TD et al. Automated analysis of genomic sequences facilitates high-throughput and comprehensive description of bacteria. ISME Communications. 2021;1DOI: 10.1038/s43705-021-00017-z

21. Lindley J. An Introduction to Botany. Longman, Orme, Brown, Green, and Longmans; 1839

22. Johnston G. Illustrations in British Zoology. Magazine of natural history and journal of zoology, 
botany, mineralogy, geology and meteorology. 1833;6:233-235.

23. de Candolle A. Lois de la Nomenclature Botanique. Masson; 1867

24. Kearfott WD. New North American Tortricidae. Transactions of the American Entomological Society. 1907;33:1-98.

25. Rawson PF, Rushton AWA, Simpson MI. Raymond Charles Casey 10 October 1917 - 26 April 2016. Biogr. Mems Fell. R. Soc. 2020;68:71-86. DOI: 10.1098/rsbm.2019.0050

26. Chomsky N, Halle M. The Sound Pattern of English. MIT Press (MA); 470: 1968

27. Hayes B. Comparative phonotactics. 2016. p. 265-285.

28. Murray AE, Freudenstein J, Gribaldo S, Hatzenpichler R, Hugenholtz $\mathbf{P}$ et al. Roadmap for naming uncultivated Archaea and Bacteria. Nat Microbiol. 2020;5:987-994. DOI: 10.1038/s41564020-0733-x

29. Konings F, Perkins MD, Kuhn JH, Pallen MJ, Alm EJ et al. SARS-CoV-2 Variants of Interest and Concern naming scheme conducive for global discourse. Nat Microbiol. 2021;6:821-823. DOI: 10.1038/s41564-021-00932-w 10.1038/s41564-020-0770-5 